\title{
Research Article \\ Strong Convergence Theorem for Equilibrium Problems and Fixed Points of a Nonspreading Mapping in Hilbert Spaces
}

\author{
Somyot Plubtieng and Sukanya Chornphrom \\ Department of Mathematics, Faculty of Science, Naresuan University, Phitsanulok 65000, Thailand \\ Correspondence should be addressed to Somyot Plubtieng, somyotp@nu.ac.th
}

Received 30 June 2010; Revised 10 October 2010; Accepted 13 December 2010

Academic Editor: Brailey Sims

Copyright (C) 2010 S. Plubtieng and S. Chornphrom. This is an open access article distributed under the Creative Commons Attribution License, which permits unrestricted use, distribution, and reproduction in any medium, provided the original work is properly cited.

We introduce an iterative method for finding a common element of the set of solutions of equilibrium problems and the set of fixed points of a nonspreading mapping in a Hilbert space. Then, we prove a strong convergence theorem which is connected with the work of S. Takahashi and W. Takahashi (2007) and Iemoto and Takahashi (2009).

\section{Introduction}

Let $H$ be a real Hilbert space with inner product $\langle\cdot, \cdot\rangle$ and norm $\|\cdot\|$, respectively, and let $C$ be a closed convex subset of $H$. Let $F: C \times C \rightarrow \mathbb{R}$ be bifunction, where $\mathbb{R}$ is the set of real numbers. The equilibrium problem for $F: C \times C \rightarrow \mathbb{R}$ is to find $x \in C$ such that

$$
F(x, y) \geq 0 \quad \forall y \in C
$$

The set of solution of (1.1) is denoted by $\mathrm{EP}(F)$. Given a mapping $A: C \rightarrow H$, let $F(x, y)=\langle A x, y-x\rangle$ for all $x, y \in C$. Then, $z \in \operatorname{EP}(F)$ if and only if $\langle A z, y-z\rangle \geq 0$ for all $y \in C$, that is, $z$ is a solution of the variational inequality. Numerous problems in physics, optimization, and economics reduce to find a solution of (1.1); see, for example, [1-9] and the references therein.

A mapping $T$ of $C$ into itself is said to be nonexpansive if $\|T x-T y\| \leq\|x-y\|$ for all $x, y \in C$, and a mapping $F$ is said to be firmly nonexpansive if $\|F x-F y\|^{2} \leq\langle x-y, F x-F y\rangle$ for all $x, y \in C$. Let $E$ be a smooth, strictly convex and reflexive Banach space, and let $J$ be the 
duality mapping of $E$ and $C$ a nonempty closed convex subset of $E$. A mapping $S: C \rightarrow C$ is said to be nonspreading if

$$
\phi(S x, S y)+\phi(S y, S x) \leq \phi(S x, y)+\phi(S y, x)
$$

for all $x, y \in C$, where $\phi(x, y)=\|x\|^{2}-2\langle x, J y\rangle+\|y\|^{2}$ for all $x, y \in E$; see, for instance, Kohsaka and Takahashi [10]. In the case when $E$ is a Hilbert space, we know that $\phi(x, y)=\|x-y\|^{2}$ for all $x, y \in E$. Then a nonspreading mapping $S: C \rightarrow C$ in a Hilbert space $H$ is defined as follows:

$$
2\|S x-S y\|^{2} \leq\|S x-y\|^{2}+\|x-S y\|^{2}
$$

for all $x, y \in C$. Let $F(Q)$ be the set of fixed points of $Q$, and $F(Q)$ nonempty; a mapping $Q: C \rightarrow C$ is said to be quasi-nonexpansive if $\|Q x-y\| \leq\|x-y\|$ for all $x \in C$ and $y \in F(Q)$.

Remark 1.1. In a Hilbert space, we know that every firmly nonexpansive mapping is nonspreading and that if the set of fixed points of a nonspreading mapping is nonempty, the nonspreading mapping is quasi-nonexpansive; see [10,11].

In 1953, Mann [12] introduced the iteration as follows: a sequence $\left\{x_{n}\right\}$ defined by

$$
x_{n+1}=\alpha_{n} x_{n}+\left(1-\alpha_{n}\right) T x_{n}
$$

where the initial guess element $x_{0} \in C$ is arbitrary and $\left\{\alpha_{n}\right\}$ is a real sequence in $[0,1]$. Mann iteration has been extensively investigated for nonexpansive mappings. In an infinite-dimensional Hilbert space, Mann iteration can conclude only weak convergence (see $[12,13])$. Fourteen years later, Halpern [14] introduced the following iterative scheme for approximating a fixed point of $T$ :

$$
x_{n+1}=\alpha_{n} x+\left(1-\alpha_{n}\right) T x_{n}
$$

for all $n \in \mathbb{N}$, where $x_{1}=x \in C$ and $\left\{\alpha_{n}\right\}$ is a sequence of $[0,1]$. Strong convergence of this type iterative sequence has been widely studied: Wittmann [15] discussed such a sequence in a Hilbert space.

On the other hand, Kohsaka and Takahashi [10] proved an existence theorem of fixed point for nonspreading mappings in a Banach space. Recently, Lemoto and Takahashi [16] studied the approximation theorem of common fixed points for a nonexpansive mapping $T$ of $C$ into itself and a nonspreading mapping $S$ of $C$ into itself in a Hilbert space. In particular, this result reduces to approximation fixed points of a nonspreading mapping $S$ of $C$ into itself in a Hilbert space by using iterative scheme

$$
x_{n+1}=\alpha_{n} x_{n}+\left(1-\alpha_{n}\right) S x_{n} .
$$

Some methods have been proposed to solve the equilibrium problem and fixed point problem of nonexpansive mapping: see, for instance, $[1,2,6,7,17-20]$ and the references 
therein. In 1997, Combettes and Hirstoaga [3] introduced an iterative scheme of finding the best approximation to the initial data when $\mathrm{EP}(F)$ is nonempty and proved a strong convergence theorem. Recently, S. Takahashi and W. Takahashi [8] introduced an iterative scheme by the viscosity approximation method for finding a common element of the set of solution of equilibrium problems and the set of fixed points of a nonexpansive mapping in a Hilbert space. Let $S: C \rightarrow H$ be a nonexpansive mapping. In 2008, Plubtieng and Punpaeng [7] introduced a new iterative sequence for finding a common element of the set of solution of equilibrium problems and the set of fixed points of a nonexpansive mapping in a Hilbert space which is the optimality condition for the minimization problem. Very recently, S. Takahashi and W. Takahashi [9] introduced an iterative method for finding a common element of the set of solutions of a generalized equilibrium problem and the set of fixed points of a nonexpansive mapping in a Hilbert space and then obtain that the sequence converges strongly to a common element of two sets.

In this paper, motivated by S. Takahashi and W. Takahashi [8] and Lemoto and Takahashi [16], we introduce an iterative sequence and prove a strong convergence theorem for finding solution of equilibrium problems and the set of fixed points of a nonspreading mapping in Hilbert spaces.

\section{Preliminaries}

Let $H$ be a real Hilbert space. When $\left\{x_{n}\right\}$ is a sequence in $H, x_{n} \rightarrow x$ implies that $x_{n}$ converges weakly to $x$ and $x_{n} \rightarrow x$ means the strong convergence. Let $C$ be a nonempty closed convex subset of $H$. For every point $x \in H$, there exists a unique nearest point in $C$; denote by $P_{C} x$, such that

$$
\left\|x-P_{C} x\right\| \leq\|x-y\| \quad \forall y \in C .
$$

$P_{C}$ is called the metric projection of $H$ onto $C$. We know that $P_{C}$ is nonexpansive. Further, for $x \in H$ and $z \in C$,

$$
z=P_{C} x \Longleftrightarrow\langle x-z, z-y\rangle \geq 0 \quad \forall y \in C
$$

Moreover, $P_{C} x$ is characterized by the following properties: $P_{C} x \in C$ and

$$
\begin{gathered}
\left\langle x-P_{C} x, y-P_{C} y\right\rangle \leq 0, \\
\|x-y\|^{2} \geq\left\|x-P_{C} x\right\|^{2}+\left\|y-P_{C} x\right\|^{2}
\end{gathered}
$$

for all $x \in H, y \in C$. We also know that $H$ satisfies Opial's condition [21], that is, for any sequence $\left\{x_{n}\right\} \subset H$ with $x_{n} \rightarrow x$, the inequality

$$
\liminf _{n \rightarrow \infty}\left\|x_{n}-x\right\|<\liminf _{n \rightarrow \infty}\left\|x_{n}-y\right\|
$$

holds for every $y \in H$ with $x \neq y$; see [21,22] for more details. 
The following lemmas will be useful for proving the convergence result of this paper.

Lemma 2.1 (see [23]). Let $(E,\langle\cdot, \cdot\rangle)$ be an inner product space. Then for all $x, y, z \in E$ and $\alpha, \beta, \gamma \in$ $[0,1]$ with $\alpha+\beta+\gamma=1$, one has

$$
\|\alpha x+\beta y+\gamma z\|^{2}=\alpha\|x\|^{2}+\beta\|y\|^{2}+\gamma\|z\|^{2}-\alpha \beta\|x-y\|^{2}-\alpha \gamma\|x-z\|^{2}-\beta \gamma\|y-z\|^{2} .
$$

Lemma 2.2 (see [10]). Let $H$ be a Hilbert space, $C$ a nonempty closed convex subset of $H$. Let $S$ be a nonspreading mapping of $C$ into itself. Then the following are equivalent.

(1) There exists $x \in C$ such that $\left\{S^{n} x\right\}$ is bounded;

(2) $F(S)$ is nonempty.

Lemma 2.3 (see [10]). Let $H$ be a Hilbert space, $C$ a nonempty closed convex subset of $H$. Let $S$ be a nonspreading mapping of $C$ into itself. Then $F(S)$ is closed and convex.

Lemma 2.4. Let $H$ be a real Hilbert space. Then for all $x, y \in H$,

(1) $\|x+y\|^{2} \leq\|x\|^{2}+2\langle y, x+y\rangle$

(2) $\|x+y\|^{2} \geq\|x\|^{2}+2\langle y, x\rangle$.

Lemma 2.5 (see [24]). Let $\left\{a_{n}\right\},\left\{b_{n}\right\} \subset[0, \infty)$, and let $\left\{c_{n}\right\} \subset[0,1)$ be sequences of real numbers such that

$$
\begin{aligned}
& a_{n+1} \leq\left(1-c_{n}\right) a_{n}+b_{n}, \text { for all } n \in \mathbb{N}, \\
& \sum_{n=1}^{\infty} c_{n}=\infty \text { and } \sum_{n=1}^{\infty} b_{n}<\infty .
\end{aligned}
$$

Then, $\lim _{n \rightarrow \infty} a_{n}=0$.

Lemma 2.6 (see [16]). Let $H$ be a Hilbert space, $C$ a closed convex subset of $H$, and $S: C \rightarrow C$ a nonspreading mapping with $F(S) \neq \emptyset$. Then $S$ is demiclosed, that is, $x_{n} \rightarrow u$ and $x_{n}-S x_{n} \rightarrow 0$ imply $u \in F(S)$.

Lemma 2.7 (see [16]). Let $H$ be a Hilbert space, $C$ a nonempty closed convex subset of a real Hilbert space $H$, and let $S$ be a nonspreading mapping of $C$ into itself, and let $A=I-S$. Then

$$
\|A x-A y\|^{2} \leq\langle x-y, A x-A y\rangle+\frac{1}{2}\left(\|A x\|^{2}+\|A y\|^{2}\right) .
$$

Lemma 2.8 (see [25]). Assume $\left\{a_{n}\right\}$ is a sequence of nonnegative real numbers such that

$$
a_{n+1} \leq\left(1-\alpha_{n}\right) a_{n}+\delta_{n}, \quad n \geq 0,
$$

where $\left\{\alpha_{n}\right\}$ is a sequence in $(0,1)$ and $\left\{\delta_{n}\right\}$ is a sequence in $\mathbb{R}$ such that

(1) $\sum_{n=1}^{\infty} \alpha_{n}=\infty$;

(2) $\lim \sup _{n \rightarrow \infty}\left(\delta_{n} / \alpha_{n}\right) \leq 0$ or $\sum_{n=1}^{\infty}\left|\delta_{n}\right|<\infty$.

Then $\lim _{n \rightarrow \infty} a_{n}=0$. 
For solving the equilibrium problems for a bifunction $F: C \times C \rightarrow \mathbb{R}$, let us assume that $F$ satisfies the following conditions:

(A1) $F(x, x)=0$ for all $x \in C$;

(A2) $F$ is monotone, that is, $F(x, y)+F(y, x) \leq 0$ for all $x, y \in C$;

(A3) for each $x, y, z \in C, \lim _{t \downarrow} F(t z+(1-t) x, y) \leq F(x, y)$;

(A4) for each $x \in C, y \mapsto F(x, y)$ is convex and lower semicontinuous.

The following lemma appears implicitly in [26].

Lemma 2.9 (see [26]). Let $C$ be a nonempty closed convex subset of $H$, and let $F$ be a bifunction of $C \times C$ into $\mathbb{R}$ satisfying (A1)-(A4). Let $r>0$ and $x \in H$. Then, there exists $z \in C$ such that

$$
F(z, y)+\frac{1}{r}\langle y-z, z-x\rangle \geq 0 \quad \forall y \in C
$$

The following lemma was also given in [4].

Lemma 2.10 (see [4]). Assume that $F: C \times C \rightarrow \mathbb{R}$ satisfies (A1)-(A4). For $r>0$ and $x \in H$, define a mapping $T_{r}: H \rightarrow C$ as follows:

$$
T_{r}(x)=\left\{z \in C: F(z, y)+\frac{1}{r}\langle y-z, z-x\rangle \geq 0, \forall y \in C\right\}
$$

for all $z \in H$. Then, the following hold:

(1) $T_{r}$ is single-valued;

(2) $T_{r}$ is firmly nonexpansive, that is, for any $x, y \in H,\left\|T_{r} x-T_{r} y\right\|^{2} \leq\left\langle T_{r} x-T_{r} y, x-y\right\rangle$;

(3) $F\left(T_{r}\right)=E P(F)$;

(4) $E P(F)$ is closed and convex.

Lemma 2.11 (see [27]). Let $\left(\Gamma_{n}\right)$ be a sequence of real numbers that does not decrease at infinity, in the sense that there exists a subsequence $\left(\Gamma_{n_{j}}\right)_{j \geq 0}$ of $\left(\Gamma_{n}\right)$ which satisfies $\Gamma_{n_{j}}<\Gamma_{n_{j}+1}$ for all $j \geq 0$. Also consider the sequence of integers $(\tau(n))_{n \geq n_{0}}$ defined by

$$
\tau(n)=\max \left\{k \leq n \mid \Gamma_{k}<\Gamma_{k+1}\right\} .
$$

Then $(\tau(n))_{n \geq n_{0}}$ is a nondecreasing sequence verifying $\lim _{n \rightarrow \infty} \tau(n)=\infty$, and the following properties are satisfied for all $n \geq n_{0}$ :

$$
\Gamma_{\tau(n)} \leq \Gamma_{\tau(n)+1}, \quad \Gamma_{n} \leq \Gamma_{\tau(n)+1} .
$$

\section{Main Result}

In this section, we prove a strong convergence theorem for finding a common element of the set of fixed points of a nonspreading mapping and the set of solutions of the equilibrium problems. 
Theorem 3.1. Let $C$ be a nonempty closed convex subset of a real Hilbert space $H$. Let $F$ be a bifunctions from $C \times C \rightarrow \mathbb{R}$ satisfying (A1)-(A4), and let $S$ be a nonspreading mapping of $C$ into itself such that $F(S) \cap E P(F) \neq \emptyset$. Let $u \in C$, and let $\left\{x_{n}\right\}$ and $\left\{u_{n}\right\}$ be sequences generated by $x_{1} \in C$ and

$$
\begin{gathered}
F\left(u_{n}, y\right)+\frac{1}{r_{n}}\left\langle y-u_{n}, u_{n}-x_{n}\right\rangle \geq 0, \quad \forall y \in C, \\
x_{n+1}=\beta_{n} x_{n}+\left(1-\beta_{n}\right) S\left[\alpha_{n} u+\left(1-\alpha_{n}\right) u_{n}\right],
\end{gathered}
$$

for all $n \in \mathbb{N}$, where $\left\{\alpha_{n}\right\},\left\{\beta_{n}\right\} \in[0,1]$ and $\left\{r_{n}\right\} \in(0, \infty)$ satisfy

$$
\begin{aligned}
& \lim _{n \rightarrow \infty} \alpha_{n}=0, \sum_{n=1}^{\infty} \alpha_{n}=\infty, 0<a \leq \beta_{n} \leq b<1, \\
& \sum_{n=1}^{\infty}\left|\alpha_{n}-\alpha_{n-1}\right|<\infty, \sum_{n=1}^{\infty}\left|\beta_{n}-\beta_{n-1}\right|<\infty, \\
& \lim \inf _{n \rightarrow \infty} r_{n}>0, \text { and } \sum_{n=1}^{\infty}\left|r_{n+1}-r_{n}\right|<\infty .
\end{aligned}
$$

Then $\left\{x_{n}\right\}$ converges strongly to $z \in F(S) \cap E P(F)$, where $z=P_{F(S) \cap E P(F)} u$.

Proof. Let $p \in F(S) \cap \operatorname{EP}(F)$. From $u_{n}=T_{r_{n}} x_{n}$, we have

$$
\left\|u_{n}-p\right\|=\left\|T_{r_{n}} x_{n}-T_{r_{n}} p\right\| \leq\left\|x_{n}-p\right\|
$$

for all $n \in \mathbb{N}$. Put $y_{n}=\alpha_{n} u+\left(1-\alpha_{n}\right) u_{n}$. We divide the proof into several steps.

Step 1. We claim that the sequences $\left\{x_{n}\right\},\left\{u_{n}\right\},\left\{y_{n}\right\}$, and $\left\{S y_{n}\right\}$ are bounded. First, we note that

$$
\begin{aligned}
\left\|S y_{n}-p\right\| & \leq\left\|y_{n}-p\right\| \\
& =\left\|\alpha_{n} u+\left(1-\alpha_{n}\right) u_{n}-p\right\| \\
& \leq \alpha_{n}\|u-p\|+\left(1-\alpha_{n}\right)\left\|u_{n}-p\right\| \\
& \leq \alpha_{n}\|u-p\|+\left(1-\alpha_{n}\right)\left\|x_{n}-p\right\|,
\end{aligned}
$$

and so

$$
\begin{aligned}
\left\|x_{n+1}-p\right\| & =\left\|\beta_{n} x_{n}+\left(1-\beta_{n}\right) S y_{n}-p\right\| \\
& \leq \beta_{n}\left\|x_{n}-p\right\|+\left(1-\beta_{n}\right)\left\|S y_{n}-p\right\| \\
& \leq \beta_{n}\left\|x_{n}-p\right\|+\left(1-\beta_{n}\right)\left\|y_{n}-p\right\| \\
& =\beta_{n}\left\|x_{n}-p\right\|+\left(1-\beta_{n}\right)\left\|\alpha_{n} u+\left(1-\alpha_{n}\right) u_{n}-p\right\| \\
& \leq \beta_{n}\left\|x_{n}-p\right\|+\left(1-\beta_{n}\right)\left(\alpha_{n}\|u-p\|+\left(1-\alpha_{n}\right)\left\|u_{n}-p\right\|\right) \\
& \leq \beta_{n}\left\|x_{n}-p\right\|+\left(1-\beta_{n}\right)\left(\alpha_{n}\|u-p\|+\left(1-\alpha_{n}\right)\left\|x_{n}-p\right\|\right) \\
& =\left(1-\alpha_{n}\left(1-\beta_{n}\right)\right)\left\|x_{n}-p\right\|+\alpha_{n}\left(1-\beta_{n}\right)\|u-p\| .
\end{aligned}
$$


Putting $M=\max \left\{\left\|x_{n}-p\right\|,\|u-p\|\right\}$, we note that $\left\|x_{n}-p\right\| \leq M$ for all $n \in \mathbb{N}$. In fact, it is obvious that $\left\|x_{1}-p\right\| \leq M$. Assume that $\left\|x_{k}-p\right\| \leq M$ for all $k \in \mathbb{N}$. Thus, we have

$$
\begin{aligned}
\left\|x_{k+1}-p\right\| & \leq\left(1-\alpha_{k}\left(1-\beta_{k}\right)\right)\left\|x_{k}-p\right\|+\alpha_{k}\left(1-\beta_{k}\right)\|u-p\| \\
& \leq\left(1-\alpha_{k}\left(1-\beta_{k}\right)\right) M+\alpha_{k}\left(1-\beta_{k}\right) M \\
& =M .
\end{aligned}
$$

By induction, we obtain that $\left\|x_{n}-p\right\| \leq M$ for all $n \in \mathbb{N}$. So, $\left\{x_{n}\right\}$ is bound. Hence, $\left\{u_{n}\right\},\left\{y_{n}\right\}$, and $\left\{S y_{n}\right\}$ are also bounded.

Step 2. Put $t_{n}=\beta_{n} y_{n}+\left(1-\beta_{n}\right) S y_{n}$. We claim that $\left\|x_{n+1}-t_{n}\right\| \rightarrow 0$ as $n \rightarrow \infty$. We note that

$$
\begin{aligned}
\left\|x_{n+1}-x_{n}\right\|= & \left\|\left(\beta_{n} x_{n}+\left(1-\beta_{n}\right) S y_{n}\right)-\left(\beta_{n-1} x_{n-1}+\left(1-\beta_{n-1}\right) S y_{n-1}\right)\right\| \\
= & \| \beta_{n} x_{n}-\beta_{n} x_{n-1}+\beta_{n} x_{n-1}-\beta_{n-1} x_{n-1}+\left(1-\beta_{n}\right) S y_{n}-\left(1-\beta_{n}\right) S y_{n-1} \\
& +\left(1-\beta_{n}\right) S y_{n-1}-\left(1-\beta_{n-1}\right) S y_{n-1} \| \\
\leq & \beta_{n}\left\|x_{n}-x_{n-1}\right\|+\left|\beta_{n}-\beta_{n-1}\right|\left\|x_{n-1}\right\|+\left(1-\beta_{n}\right)\left\|S y_{n}-S y_{n-1}\right\| \\
& +\left|\left(1-\beta_{n}\right)-\left(1-\beta_{n-1}\right)\right|\left\|S y_{n-1}\right\| \\
\leq & \beta_{n}\left\|x_{n}-x_{n-1}\right\|+\left|\beta_{n}-\beta_{n-1}\right|\left\|x_{n-1}\right\|+\left(1-\beta_{n}\right)\left\|y_{n}-y_{n-1}\right\|+\left|\beta_{n-1}-\beta_{n}\right|\left\|S y_{n-1}\right\| \\
= & \beta_{n}\left\|x_{n}-x_{n-1}\right\|+\left|\beta_{n}-\beta_{n-1}\right|\left\|x_{n-1}\right\|+\left(1-\beta_{n}\right) \\
& \times \\
\leq & \beta_{n} \| \alpha_{n} u+\left(1-x_{n}-x_{n-1} \|+\mid \beta_{n}-\alpha_{n-1} u-\left(1-\beta_{n-1}\left|\left\|u_{n-1}\right\|+\right| \beta_{n-1}-\beta_{n-1} \mid\left\|S y_{n-1}\right\|\right.\right. \\
& \times\left[\left\|\alpha_{n} u-\alpha_{n-1} u\right\|+\|\left(1-\beta_{n}\right)\right. \\
= & \left.\beta_{n}\left\|x_{n}-x_{n-1}\right\|+\left|\beta_{n}-\beta_{n-1}\right|\left\|x_{n-1}\right\|+\left(1-\alpha_{n-1}\right) u_{n-1} \|\right]+\left|\beta_{n}-\beta_{n-1}\right|\left\|S y_{n-1}\right\| \\
& +\left(1-\beta_{n}\right)\left\|\left(1-\alpha_{n}\right) u_{n-1}-\left(1-\alpha_{n}\right) u_{n-1}+\left(1-\alpha_{n}\right) u_{n-1}-\left(1-\alpha_{n-1}\right) u_{n-1}\right\| \\
& +\left|\beta_{n}-\beta_{n-1}\right|\left\|S y_{n-1}\right\| \\
\leq & \beta_{n}\left\|x_{n}-x_{n-1}\right\|+\left|\beta_{n}-\beta_{n-1}\right|\left\|x_{n-1}\right\|+\left(1-\beta_{n}\right)\left|\alpha_{n}-\alpha_{n-1}\right|\|u\| \\
& +\left(1-\beta_{n}\right)\left(1-\alpha_{n}\right)\left\|u_{n}-u_{n-1}\right\|+\left(1-\beta_{n}\right)\left|\left(1-\alpha_{n}\right)-\left(1-\alpha_{n-1}\right)\right|\left\|u_{n-1}\right\| \\
& +\left|\beta_{n}-\beta_{n-1}\right|\left\|S y_{n-1}\right\| \\
= & \beta_{n}\left\|x_{n}-x_{n-1}\right\|+\left|\beta_{n}-\beta_{n-1}\right|\left\|x_{n-1}\right\|+\left(1-\beta_{n}\right)\left|\alpha_{n}-\alpha_{n-1}\right|\|u\| \\
& +\left(1-\beta_{n}\right)\left(1-\alpha_{n}\right)\left\|u_{n}-u_{n-1}\right\|+\left(1-\beta_{n}\right)\left|\alpha_{n}-\alpha_{n-1}\right|\left\|u_{n-1}\right\| \\
& +\left|\beta_{n}-\beta_{n-1}\right|\left\|S y_{n-1}\right\| \\
= & \beta_{n}\left\|x_{n}-x_{n-1}\right\|+\left|\beta_{n}-\beta_{n-1}\right| K_{1}+\left(1-\beta_{n}\right)\left|\alpha_{n}-\alpha_{n-1}\right| K_{1} \\
& +\left(1-\beta_{n}\right)\left(1-\alpha_{n}\right)\left\|u_{n}-u_{n-1}\right\|+\left(1-\beta_{n}\right)\left|\alpha_{n}-\alpha_{n-1}\right| K_{1}+\left|\beta_{n}-\beta_{n-1}\right| K_{1}, \\
& +(1-1)
\end{aligned}
$$


where $K_{1}=\sup \left\{\left\|x_{n}\right\|+\left\|S y_{n}\right\|+\|u\|+\left\|u_{n-1}\right\|: n \in \mathbb{N}\right\}$. On the other hand, from $u_{n}=T_{r_{n}} x_{n}$ and $u_{n+1}=T_{r_{n+1}} x_{n+1}$, we have

$$
\begin{gathered}
F\left(u_{n}, y\right)+\frac{1}{r_{n}}\left\langle y-u_{n}, u_{n}-x_{n}\right\rangle \geq 0, \\
F\left(u_{n+1}, y\right)+\frac{1}{r_{n+1}}\left\langle y-u_{n+1}, u_{n+1}-x_{n+1}\right\rangle \geq 0
\end{gathered}
$$

for all $y \in C$. Putting $y=u_{n+1}$ in (3.7) and $y=u_{n}$ in (3.8), we have

$$
\begin{gathered}
F\left(u_{n}, u_{n+1}\right)+\frac{1}{r_{n}}\left\langle u_{n+1}-u_{n}, u_{n}-x_{n}\right\rangle \geq 0, \\
F\left(u_{n+1}, u_{n}\right)+\frac{1}{r_{n+1}}\left\langle u_{n}-u_{n+1}, u_{n+1}-x_{n+1}\right\rangle \geq 0 .
\end{gathered}
$$

So, from (A2), we note that

$$
\left\langle u_{n+1}-u_{n}, \frac{u_{n}-x_{n}}{r_{n}}-\frac{u_{n+1}-x_{n+1}}{r_{n+1}}\right\rangle \geq 0
$$

and hence

$$
\left\langle u_{n+1}-u_{n}, u_{n}-u_{n+1}+u_{n+1}-x_{n}-\frac{r_{n}}{r_{n+1}}\left(u_{n+1}-x_{n+1}\right)\right\rangle \geq 0
$$

Without loss of generality, let us assume that there exists a real number $d$ such that $r_{n}>d>0$ for all $n \in \mathbb{N}$. Thus, we have

$$
\begin{aligned}
\left\|u_{n+1}-u_{n}\right\|^{2} & \leq\left\langle u_{n+1}-u_{n}, x_{n+1}-x_{n}+\left(1-\frac{r_{n}}{r_{n+1}}\right)\left(u_{n+1}-x_{n+1}\right)\right\rangle \\
& \leq\left\|u_{n+1}-u_{n}\right\|\left\{\left\|x_{n+1}-x_{n}\right\|+\left|1-\frac{r_{n}}{r_{n+1}}\right|\left\|u_{n+1}-x_{n+1}\right\|\right\},
\end{aligned}
$$

and hence

$$
\begin{aligned}
\left\|u_{n+1}-u_{n}\right\| & \leq\left\|x_{n+1}-x_{n}\right\|+\frac{1}{r_{n+1}}\left|r_{n+1}-r_{n}\right||| u_{n+1}-x_{n+1} \| \\
& \leq\left\|x_{n+1}-x_{n}\right\|+\frac{1}{d}\left|r_{n+1}-r_{n}\right| L
\end{aligned}
$$


where $L=\sup \left\{\left\|u_{n}-x_{n}\right\|: n \in \mathbb{N}\right\}$. So, from (3.6), we note that

$$
\begin{aligned}
\left\|x_{n+1}-x_{n}\right\| \leq & \beta_{n}\left\|x_{n}-x_{n-1}\right\|+2\left|\beta_{n}-\beta_{n-1}\right| K_{1}+2\left(1-\beta_{n}\right)\left|\alpha_{n}-\alpha_{n-1}\right| K_{1} \\
& +\left(1-\beta_{n}\right)\left(1-\alpha_{n}\right)\left(\left\|x_{n}-x_{n-1}\right\|+\frac{1}{d}\left|r_{n}-r_{n-1}\right| L\right) \\
= & \left(\beta_{n}+\left(1-\beta_{n}\right)\left(1-\alpha_{n}\right)\right)\left\|x_{n}-x_{n-1}\right\|+2\left|\beta_{n}-\beta_{n-1}\right| K_{1}+2\left(1-\beta_{n}\right)\left|\alpha_{n}-\alpha_{n-1}\right| K_{1} \\
& +\left(1-\beta_{n}\right)\left(1-\alpha_{n}\right) \frac{1}{d}\left|r_{n}-r_{n-1}\right| L \\
= & \left(1-\left(1-\beta_{n}\right) \alpha_{n}\right)\left\|x_{n}-x_{n-1}\right\|+2\left|\beta_{n}-\beta_{n-1}\right| K_{1}+2\left(1-\beta_{n}\right)\left|\alpha_{n}-\alpha_{n-1}\right| K_{1} \\
& +\left(1-\beta_{n}\right)\left(1-\alpha_{n}\right) \frac{L}{d}\left|r_{n}-r_{n-1}\right| .
\end{aligned}
$$

By Lemma 2.5, we have

$$
\lim _{n \rightarrow \infty}\left\|x_{n+1}-x_{n}\right\|=0
$$

for $p \in F(S) \cup \operatorname{EP}(F)$. We note from $u_{n}=T_{r_{n}} x_{n}$ that

$$
\begin{aligned}
\left\|u_{n}-p\right\|^{2} & =\left\|T_{r_{n}} x_{n}-T_{r_{n}} p\right\|^{2} \leq\left\langle T_{r_{n}} x_{n}-T_{r_{n}} p, x_{n}-p\right\rangle=\left\langle u_{n}-p, x_{n}-p\right\rangle \\
& =\frac{1}{2}\left(\left\|u_{n}-p\right\|^{2}+\left\|x_{n}-p\right\|^{2}-\left\|x_{n}-u_{n}\right\|^{2}\right),
\end{aligned}
$$

and hence

$$
\left\|u_{n}-p\right\|^{2} \leq\left\|x_{n}-p\right\|^{2}-\left\|x_{n}-u_{n}\right\|^{2} .
$$

Therefore, from the convexity of $\|\cdot\|^{2}$, we have

$$
\begin{aligned}
\left\|x_{n+1}-p\right\|^{2} & =\left\|\beta_{n} x_{n}+\left(1-\beta_{n}\right) S y_{n}-p\right\|^{2} \\
& \leq \beta_{n}\left\|x_{n}-p\right\|^{2}+\left(1-\beta_{n}\right)\left\|S y_{n}-p\right\|^{2} \\
& \leq \beta_{n}\left\|x_{n}-p\right\|^{2}+\left(1-\beta_{n}\right)\left\|y_{n}-p\right\|^{2} \\
& =\beta_{n}\left\|x_{n}-p\right\|^{2}+\left(1-\beta_{n}\right)\left\|\alpha_{n} u+\left(1-\alpha_{n}\right) u_{n}-p\right\|^{2} \\
& \leq \beta_{n}\left\|x_{n}-p\right\|^{2}+\alpha_{n}\left(1-\beta_{n}\right)\|u-p\|^{2}+\left(1-\beta_{n}\right)\left(1-\alpha_{n}\right)\left\|u_{n}-p\right\|^{2} \\
& \leq \beta_{n}\left\|x_{n}-p\right\|^{2}+\alpha_{n}\left(1-\beta_{n}\right)\|u-p\|^{2}+\left(1-\beta_{n}\right)\left(1-\alpha_{n}\right)\left(\left\|x_{n}-p\right\|^{2}-\left\|x_{n}-u_{n}\right\|^{2}\right) \\
& =\left(1-\left(1-\beta_{n}\right) \alpha_{n}\right)\left\|x_{n}-p\right\|^{2}+\alpha_{n}\left(1-\beta_{n}\right)\|u-p\|^{2}+\left(1-\beta_{n}\right)\left(1-\alpha_{n}\right)\left\|x_{n}-u_{n}\right\|^{2},
\end{aligned}
$$


and hence

$$
\begin{aligned}
\left(1-\beta_{n}\right)\left(1-\alpha_{n}\right)\left\|x_{n}-u_{n}\right\|^{2} \leq & \alpha_{n}\left(1-\beta_{n}\right)\|u-p\|^{2}-\alpha_{n}\left(1-\beta_{n}\right)\left\|x_{n}-p\right\|^{2} \\
& +\left\|x_{n}-p\right\|^{2}-\left\|x_{n+1}-p\right\|^{2} \\
= & \alpha_{n}\left(1-\beta_{n}\right)\|u-p\|^{2}-\alpha_{n}\left(1-\beta_{n}\right)\left\|x_{n}-p\right\|^{2} \\
& +\left(\left\|x_{n}-p\right\|-\left\|x_{n+1}-p\right\|\right)\left(\left\|x_{n}-p\right\|+\left\|x_{n+1}-p\right\|\right) \\
\leq & \alpha_{n}\left(1-\beta_{n}\right)\|u-p\|^{2}-\alpha_{n}\left(1-\beta_{n}\right)\left\|x_{n}-p\right\|^{2} \\
& +\left\|x_{n}-x_{n+1}\right\|\left(\left\|x_{n}-p\right\|+\left\|x_{n+1}-p\right\|\right) .
\end{aligned}
$$

So, we have $\left\|x_{n}-u_{n}\right\| \rightarrow 0$. Indeed, since $y_{n}=\alpha_{n} u+\left(1-\alpha_{n}\right) u_{n}$, it follows that

$$
\begin{aligned}
\lim _{n \rightarrow \infty}\left\|x_{n}-y_{n}\right\| & =\lim _{n \rightarrow \infty}\left\|x_{n}-\left(\alpha_{n} u+\left(1-\alpha_{n}\right) u_{n}\right)\right\| \\
& =\lim _{n \rightarrow \infty}\left\|\left(\alpha_{n}+\left(1-\alpha_{n}\right)\right) x_{n}-\left(\alpha_{n} u+\left(1-\alpha_{n}\right) u_{n}\right)\right\| \\
& \leq \lim _{n \rightarrow \infty}\left[\alpha_{n}\left\|x_{n}-u\right\|+\left(1-\alpha_{n}\right)\left\|x_{n}-u_{n}\right\|\right] \\
& =\lim _{n \rightarrow \infty} \alpha_{n}\left\|x_{n}-u\right\|+\lim _{n \rightarrow \infty}\left(1-\alpha_{n}\right)\left\|x_{n}-u_{n}\right\| \\
& =0 .
\end{aligned}
$$

Then, we note that

$$
\begin{aligned}
\left\|x_{n+1}-t_{n}\right\| & =\left\|\left(\beta_{n} x_{n}+\left(1-\beta_{n}\right) S y_{n}\right)-\left(\beta_{n} y_{n}+\left(1-\beta_{n}\right) S y_{n}\right)\right\| \\
& =\left\|\beta_{n}\left(x_{n}-y_{n}\right)+\left(1-\beta_{n}\right)\left(S y_{n}-S y_{n}\right)\right\| \\
& =\beta_{n}\left\|x_{n}-y_{n}\right\| .
\end{aligned}
$$

Since, $0<a \leq \beta_{n} \leq b<1$ and $\left\|x_{n}-y_{n}\right\| \rightarrow 0$, it follows that

$$
\lim _{n \rightarrow \infty}\left\|x_{n+1}-t_{n}\right\|=0
$$

Step 3. Put $A=I-S$. From $A p=0$, it follows by Lemma 2.7 that

$$
\begin{aligned}
\left\|t_{n}-p\right\|^{2} & =\left\|\left(\beta_{n} y_{n}+\left(1-\beta_{n}\right) S y_{n}\right)-p\right\|^{2} \\
& =\left\|\left(y_{n}-p\right)-\left(1-\beta_{n}\right)\left(y_{n}-S y_{n}\right)\right\|^{2} \\
& =\left\|\left(y_{n}-p\right)-\left(1-\beta_{n}\right) A y_{n}\right\|^{2} \\
& =\left\|\left(y_{n}-p\right)\right\|^{2}-2\left(1-\beta_{n}\right)\left\langle y_{n}-p, A y_{n}-A p\right\rangle+\left(1-\beta_{n}\right)^{2}\left\|A y_{n}\right\|^{2}
\end{aligned}
$$


Fixed Point Theory and Applications

$$
\begin{aligned}
\leq & \left\|\left(y_{n}-p\right)\right\|^{2}-2\left(1-\beta_{n}\right)\left\{\left\|A y_{n}-A p\right\|^{2}-\frac{1}{2}\left(\left\|A y_{n}\right\|^{2}+\|A p\|^{2}\right)\right\} \\
& +\left(1-\beta_{n}\right)^{2}\left\|A y_{n}\right\|^{2} \\
= & \left\|\alpha_{n}(u-p)+\left(1-\alpha_{n}\right)\left(u_{n}-p\right)\right\|^{2}-2\left(1-\beta_{n}\right)\left\|A y_{n}\right\|^{2} \\
& +\left(1-\beta_{n}\right)\left\|A y_{n}\right\|^{2}+\left(1-\beta_{n}\right)^{2}\left\|A y_{n}\right\|^{2} \\
\leq & \alpha_{n}\|(u-p)\|^{2}+\left(1-\alpha_{n}\right)\left\|\left(u_{n}-p\right)\right\|^{2}-\beta_{n}\left(1-\beta_{n}\right)\left\|A y_{n}\right\|^{2} \\
\leq & \alpha_{n}\|(u-p)\|^{2}+\left(1-\alpha_{n}\right)\left\|\left(x_{n}-p\right)\right\|^{2}-\beta_{n}\left(1-\beta_{n}\right)\left\|A y_{n}\right\|^{2} \\
\leq & \alpha_{n}\|u-p\|^{2}+\left\|x_{n}-p\right\|^{2}-\beta_{n}\left(1-\beta_{n}\right)\left\|A y_{n}\right\|^{2} .
\end{aligned}
$$

Since $0<a \leq \beta_{n} \leq b<1$, we have $\beta_{n}\left(1-\beta_{n}\right) \geq a(1-b):=K_{2}$. Therefore, by (3.23), we obtain

$$
\begin{aligned}
K_{2}\left\|y_{n}-S y_{n}\right\|^{2} & =K_{2}\left\|A y_{n}\right\|^{2} \\
& \leq \alpha_{n}\|u-p\|^{2}+\left\|x_{n}-p\right\|^{2}-\left\|t_{n}-p\right\|^{2} \\
& \leq \alpha_{n} M^{2}+\left\|x_{n}-p\right\|^{2}-\left\|t_{n}-p\right\|^{2} \\
& =\alpha_{n} M^{2}+\left\|x_{n}-p\right\|^{2}-\left\|\left(t_{n}-x_{n+1}\right)+\left(x_{n+1}-p\right)\right\|^{2} \\
& =\alpha_{n} M^{2}+\left\|x_{n}-p\right\|^{2}-\left\|t_{n}-x_{n+1}\right\|^{2}-2\left\langle t_{n}-x_{n+1}, x_{n+1}-p\right\rangle-\left\|x_{n+1}-p\right\|^{2} \\
& \leq \alpha_{n} M^{2}+\left\|x_{n}-p\right\|^{2}-\left\|x_{n+1}-p\right\|^{2}-2\left\langle t_{n}-x_{n+1}, x_{n+1}-p\right\rangle .
\end{aligned}
$$

Step 4. Putting $z=P_{F(S) \cap E P(F)} u$, we claim that the sequence $\left\{x_{n}\right\}$ converges strongly to $z=$ $P_{F(S) \cap E P(F)} u$. Indeed, we discuss two possible cases.

Case 1. Assume that there exists $n_{0}$ such that the sequence $\left\{\left\|x_{n}-p\right\|\right\}$ is a nonincreasing sequence for all $n \geq n_{0}$. Then we have $\left\|x_{n+1}-p\right\| \leq\left\|x_{n}-p\right\|$ (for $n \geq n_{0}$ ), and hence $\lim _{n \rightarrow \infty}\left\|x_{n}-p\right\|$ exists. Therefore

$$
\lim _{n \rightarrow \infty}\left\|x_{n}-p\right\|=\lim _{n \rightarrow \infty}\left\|x_{n+1}-p\right\|
$$

By (3.22), (3.24), and (3.25), we get

$$
\left\|y_{n}-S y_{n}\right\| \longrightarrow 0
$$

Let $\left\{y_{n_{i}}\right\}$ be a subsequence of $\left\{y_{n}\right\}$ such that

$$
\limsup _{n \rightarrow \infty}\left\langle u-z, y_{n}-z\right\rangle=\lim _{n \rightarrow \infty}\left\langle u-z, y_{n_{i}}-z\right\rangle
$$


Since $\left\{y_{n}\right\}$ is bounded, there exists a subsequence $\left\{y_{n_{i}}\right\}$ of $\left\{y_{n}\right\}$ which converges weakly to $w$. Without loss of generality, we can assume that $y_{n_{i}} \rightarrow w$. Since $C$ is closed and convex, we note that $C$ is weakly closed. So, we have $w \in C$. Since $\left\|S y_{n}-y_{n}\right\| \rightarrow 0$, it follows by Lemma 2.6 that $w \in F(S)$. From (3.27) and the property of metric projection, we have

$$
\begin{aligned}
\limsup _{n \rightarrow \infty}\left\langle u-z, y_{n}-z\right\rangle & =\lim _{n \rightarrow \infty}\left\langle u-z, y_{n_{i}}-z\right\rangle \\
& =\langle u-z, w-z\rangle \leq 0
\end{aligned}
$$

Finally, we prove that $x_{n} \rightarrow z$. In fact, since $y_{n}-z=\alpha_{n}(u-z)+\left(1-\alpha_{n}\right)\left(u_{n}-z\right)$, it follows that

$$
\begin{aligned}
\left\|x_{n+1}-z\right\|^{2} & =\left\|\left(\beta_{n} x_{n}+\left(1-\beta_{n}\right) S y_{n}\right)-z\right\|^{2} \\
& \leq \beta_{n}\left\|x_{n}-z\right\|^{2}+\left(1-\beta_{n}\right)\left\|S y_{n}-z\right\|^{2} \\
& \leq \beta_{n}\left\|x_{n}-z\right\|^{2}+\left(1-\beta_{n}\right)\left\|y_{n}-z\right\|^{2} \\
& \leq \beta_{n}\left\|x_{n}-z\right\|^{2}+\left(1-\beta_{n}\right)\left[\left(1-\alpha_{n}\right)^{2}\left\|x_{n}-z\right\|^{2}+2 \alpha_{n}\left\langle u-z, y_{n}-z\right\rangle\right] \\
& \leq \beta_{n}\left\|x_{n}-z\right\|^{2}+\left(1-\beta_{n}\right)\left(1-\alpha_{n}\right)\left\|x_{n}-z\right\|^{2}+2 \alpha_{n}\left(1-\beta_{n}\right)\left\langle u-z, y_{n}-z\right\rangle \\
& =\left(1-\alpha_{n}\left(1-\beta_{n}\right)\right)\left\|x_{n}-z\right\|^{2}+2 \alpha_{n}\left(1-\beta_{n}\right)\left\langle u-z, y_{n}-z\right\rangle .
\end{aligned}
$$

By (3.28) and $\sum_{n=1}^{\infty} \alpha_{n}=\infty$, we immediately deduce by Lemma 2.8 that $x_{n} \rightarrow z$.

Case 2. Assume that for all $n \in \mathbb{N}$, there exits $m \geq n$ such that $\left\|x_{m}-p\right\|<\left\|x_{m+1}-p\right\|$. Put $a_{m}=:\left\|x_{m}-p\right\|$ for all $m \in \mathbb{N}$. Thus, it follows that there exists a subsequence $\left(a_{n_{k}}\right)_{k \geq 1}$ of $\left(a_{n}\right)_{n \geq 1}$ such that $a_{n_{k}}<a_{n_{k+1}}$ for all $k \in \mathbb{N}$. Let $\varphi: \mathbb{N}_{1} \rightarrow \mathbb{N}$ be a mapping defined by

$$
\varphi(n)=\max \left\{k \leq n: a_{k} \leq a_{k+1}\right\},
$$

where $\mathbb{N}_{1}=\left\{n \in \mathbb{N}: n \geq n_{1}\right\}$. By Lemma 2.11, we note that $\varphi(n)$ is a nondecreasing sequence such that $\varphi(n) \rightarrow \infty$ as $n \rightarrow \infty$ and that the following properties are satisfied by all numbers $n \geq n_{1}$ :

$$
a_{\varphi(n)} \leq a_{\varphi(n)+1}, \quad a_{n} \leq a_{\varphi(n)+1}
$$

From (3.24), we have

$$
\begin{aligned}
K_{2}\left\|y_{\varphi(n)}-S y_{\varphi(n)}\right\|^{2} \leq & \alpha_{\varphi(n)} M^{2}+\left\|x_{\varphi(n)}-p\right\|^{2}-\left\|x_{\varphi(n)+1}-p\right\|^{2} \\
& -2\left\langle t_{\varphi(n)}-x_{\varphi(n)+1}, x_{\varphi(n)+1}-p\right\rangle \\
\leq & \alpha_{\varphi(n)} M^{2}-2\left\langle t_{\varphi(n)}-x_{\varphi(n)+1}, x_{\varphi(n)+1}-p\right\rangle .
\end{aligned}
$$


This implies that

$$
\left\|y_{\varphi(n)}-S y_{\varphi(n)}\right\| \longrightarrow 0
$$

Take a subsequence $\left\{y_{\varphi(n)_{i}}\right\}$ of $\left\{y_{\varphi(n)}\right\}$ such that

$$
\limsup _{n \rightarrow \infty}\left\langle u-z, y_{\varphi(n)}-z\right\rangle=\lim _{n \rightarrow \infty}\left\langle u-z, y_{\varphi(n)_{i}}-z\right\rangle .
$$

From the boundedness of $\left\{y_{\varphi(n)_{i}}\right\}$, we can assume that $y_{\varphi(n)_{i}} \rightarrow v$. Since $C$ is closed and convex, it follows that $C$ is weakly closed. So, we have $v \in C$. Since $\left\|S y_{\varphi(n)}-y_{\varphi(n)}\right\| \rightarrow 0$, it follows by Lemma 2.6 that $v \in F(S)$. From (3.34) and the property of metric projection, we have

$$
\begin{aligned}
\limsup _{n \rightarrow \infty}\left\langle u-z, y_{\varphi(n)}-z\right\rangle & =\lim _{n \rightarrow \infty}\left\langle u-z, y_{\varphi(n)_{i}}-z\right\rangle \\
& =\langle u-z, v-z\rangle \\
& \leq 0 .
\end{aligned}
$$

By the same argument as (3.29) in Case 1, we conclude immediately that, for all $n \geq 1$,

$$
\begin{aligned}
0 \leq & \left\|x_{\varphi(n)+1}-z\right\|^{2}-\left\|x_{\varphi(n)}-z\right\|^{2} \\
\leq & \beta_{\varphi(n)}\left\|x_{\varphi(n)}-z\right\|^{2}+\left(1-\beta_{\varphi(n)}\right)\left\|S y_{\varphi(n)}-z\right\|^{2}-\left\|x_{\varphi(n)}-z\right\|^{2} \\
\leq & \beta_{\varphi(n)}\left\|x_{\varphi(n)}-z\right\|^{2}+\left(1-\beta_{\varphi(n)}\right)\left\|y_{\varphi(n)}-z\right\|^{2}-\left\|x_{\varphi(n)}-z\right\|^{2} \\
\leq & \beta_{\varphi(n)}\left\|x_{\varphi(n)}-z\right\|^{2}+\left(1-\beta_{\varphi(n)}\right) \\
& \times\left[\left(1-\alpha_{\varphi(n)}\right)^{2}\left\|u_{\varphi(n)}-z\right\|^{2}+2 \alpha_{\varphi(n)}\left\langle u-z, y_{\varphi(n)}-z\right\rangle\right]-\left\|x_{\varphi(n)}-z\right\|^{2} \\
\leq & \beta_{\varphi(n)}\left\|x_{\varphi(n)}-z\right\|^{2}+\left(1-\beta_{\varphi(n)}\right)\left(1-\alpha_{\varphi(n)}\right)\left\|u_{\varphi(n)}-z\right\|^{2} \\
& +2 \alpha_{\varphi(n)}\left(1-\beta_{\varphi(n)}\right)\left\langle u-z, y_{\varphi(n)}-z\right\rangle-\left\|x_{\varphi(n)}-z\right\|^{2} \\
\leq & \beta_{\varphi(n)}\left\|x_{\varphi(n)}-z\right\|^{2}+\left(1-\beta_{\varphi(n)}\right)\left(1-\alpha_{\varphi(n)}\right)\left\|x_{\varphi(n)}-z\right\|^{2} \\
& +2 \alpha_{\varphi(n)}\left(1-\beta_{\varphi(n)}\right)\left\langle u-z, y_{\varphi(n)}-z\right\rangle-\left\|x_{\varphi(n)}-z\right\|^{2} \\
= & \alpha_{\varphi(n)}\left(1-\beta_{\varphi(n)}\right)\left[2\left\langle u-z, y_{\varphi(n)}-z\right\rangle-\left\|x_{\varphi(n)}-z\right\|^{2}\right] \\
\leq & 2\left\langle u-z, y_{\varphi(n)}-z\right\rangle-\left\|x_{\varphi(n)}-z\right\|^{2},
\end{aligned}
$$

which implies that

$$
\left\|x_{\varphi(n)}-z\right\|^{2} \leq 2\left\langle u-z, y_{\varphi(n)}-z\right\rangle .
$$


By (3.35), we have

$$
\lim _{n \rightarrow \infty}\left\|x_{\varphi(n)}-z\right\|=0
$$

and hence

$$
\lim _{n \rightarrow \infty}\left\|x_{\varphi(n)+1}-z\right\|=\lim _{n \rightarrow \infty}\left\|x_{\varphi(n)}-z\right\|=0
$$

Since $\left\|x_{n}-z\right\|=a_{n} \leq a_{\varphi(n)}=\left\|x_{\varphi(n)}-z\right\|$ for all $n \geq n_{1}$, we have

$$
\lim _{n \rightarrow \infty}\left\|x_{n}-z\right\|=0
$$

This completes the proof.

As direct consequences of Theorem 3.1, we obtain corollaries.

Corollary 3.2. Let $C$ be a nonempty closed convex subset of a real Hilbert space $H$. Let $F$ be a bifunctions from $C \times C \rightarrow \mathbb{R}$ satisfying (A1)-(A4), and let $S$ be a firmly nonexpansive mapping of $C$ into itself such that $F(S) \cap E P(F) \neq \emptyset$. Let $u \in C$, and let $\left\{x_{n}\right\}$ and $\left\{u_{n}\right\}$ be sequences generated by $x_{1} \in C$ and

$$
\begin{gathered}
F\left(u_{n}, y\right)+\frac{1}{r_{n}}\left\langle y-u_{n}, u_{n}-x_{n}\right\rangle \geq 0, \quad \forall y \in C, \\
x_{n+1}=\beta_{n} x_{n}+\left(1-\beta_{n}\right) S\left[\alpha_{n} u+\left(1-\alpha_{n}\right) u_{n}\right],
\end{gathered}
$$

for all $n \in \mathbb{N}$, where $\left\{\alpha_{n}\right\},\left\{\beta_{n}\right\} \in[0,1]$ and $\left\{r_{n}\right\} \in(0, \infty)$ satisfy

$$
\begin{aligned}
& \lim _{n \rightarrow \infty} \alpha_{n}=0, \sum_{n=1}^{\infty} \alpha_{n}=\infty, 0<a \leq \beta_{n} \leq b<1, \\
& \sum_{n=1}^{\infty}\left|\alpha_{n}-\alpha_{n-1}\right|<\infty, \sum_{n=1}^{\infty}\left|\beta_{n}-\beta_{n-1}\right|<\infty, \\
& \lim \inf _{n \rightarrow \infty} r_{n}>0, \text { and } \sum_{n=1}^{\infty}\left|r_{n+1}-r_{n}\right|<\infty .
\end{aligned}
$$

Then $\left\{x_{n}\right\}$ converges strongly to $z \in F(S) \cap E P(F)$, where $z=P_{F(S) \cap E P(F)} u$.

\section{Acknowledgments}

The authors would like to thank the referees for the insightful comments and suggestions. Moreover, the authors gratefully acknowledge the Thailand Research Fund Master Research Grants (TRF-MAG, MRG-WII515S029) for funding this paper.

\section{References}

[1] S.-S. Chang, H. W. Joseph Lee, and C. K. Chan, "A new method for solving equilibrium problem fixed point problem and variational inequality problem with application to optimization," Nonlinear Analysis Theory: Methods \& Applications, vol. 70, no. 9, pp. 3307-3319, 2009. 
[2] V. Colao, G. Marino, and H.-K. Xu, “An iterative method for finding common solutions of equilibrium and fixed point problems," Journal of Mathematical Analysis and Applications, vol. 344, no. 1, pp. 340352, 2008.

[3] P. L. Combettes and S. A. Hirstoaga, "Equilibrium programming using proximal-like algorithms," Mathematical Programming, vol. 78, no. 1, pp. 29-41, 1997.

[4] P. L. Combettes and S. A. Hirstoaga, "Equilibrium programming in Hilbert spaces," Journal of Nonlinear and Convex Analysis, vol. 63, pp. 123-145, 1994.

[5] J.-W. Peng, Y. Wang, D. S. Shyu, and J.-C. Yao, "Common solutions of an iterative scheme for variational inclusions, equilibrium problems, and fixed point problems," Journal of Inequalities and Applications, vol. 2008, Article ID 720371, 15 pages, 2008.

[6] S. Plubtieng and R. Punpaeng, "A new iterative method for equilibrium problems and fixed point problems of nonexpansive mappings and monotone mappings," Applied Mathematics and Computation, vol. 197, no. 2, pp. 548-558, 2008.

[7] S. Plubtieng and R. Punpaeng, "A general iterative method for equilibrium problems and fixed point problems in Hilbert spaces," Journal of Mathematical Analysis and Applications, vol. 336, no. 1, pp. 455469, 2007.

[8] S. Takahashi and W. Takahashi, "Viscosity approximation methods for equilibrium problems and fixed point problems in Hilbert spaces," Journal of Mathematical Analysis and Applications, vol. 331, no. 1, pp. 506-515, 2007.

[9] S. Takahashi and W. Takahashi, "Strong convergence theorem for a generalized equilibrium problem and a nonexpansive mapping in a Hilbert space," Nonlinear Analysis: Theory, Methods E Applications, vol. 69 , no. 3, pp. 1025-1033, 2008.

[10] F. Kohsaka and W. Takahashi, "Fixed point theorems for a class of nonlinear mappings related to maximal monotone operators in Banach spaces," Archiv der Mathematik, vol. 91, no. 2, pp. 166-177, 2008.

[11] S. Plubtieng and K. Sombut, "Weak convergence theorems for a system of mixed equilibrium problems and nonspreading mappings in a Hilbert space," Journal of Inequalities and Applications, vol. 2010, Article ID 246237, 12 pages, 2010.

[12] W. R. Mann, "Mean value methods in iteration," Proceedings of the American Mathematical Society, vol. 4, pp. 506-510, 1953.

[13] S. Reich, "Weak convergence theorems for nonexpansive mappings in Banach spaces," Journal of Mathematical Analysis and Applications, vol. 67, no. 2, pp. 274-276, 1979.

[14] B. Halpern, "Fixed points of nonexpanding maps," Bulletin of the American Mathematical Society, vol. 73, pp. 957-961, 1967.

[15] R. Wittmann, "Approximation of fixed points of nonexpansive mappings," Archiv der Mathematik, vol. 58, no. 5, pp. 486-491, 1992.

[16] S. Iemoto and W. Takahashi, "Approximating common fixed points of nonexpansive mappings and nonspreading mappings in a Hilbert space," Nonlinear Analysis: Theory, Methods \& Applications, vol. 71, no. 12, pp. e2082-e2089, 2009.

[17] S. Plubtieng and W. Sriprad, "A viscosity approximation method for finding common solutions of variational inclusions, equilibrium problems, and fixed point problems in Hilbert spaces," Fixed Point Theory and Applications, vol. 2009, Article ID 567147, 20 pages, 2009.

[18] S. Plubtieng and W. Sriprad, "Hybrid methods for equilibrium problems and fixed points problems of a countable family of relatively nonexpansive mappings in Banach spaces," Fixed Point Theory and Applications, vol. 2010, Article ID 962628, 17 pages, 2010.

[19] S. Plubtieng and T. Thammathiwat, "A viscosity approximation method for equilibrium problems, fixed point problems of nonexpansive mappings and a general system of variational inequalities," Journal of Global Optimization, vol. 46, no. 3, pp. 447-464, 2010.

[20] S. Plubtieng and T. Thammathiwat, "A viscosity approximation method for finding a common solution of fixed points and equilibrium problems in Hilbert spaces," Journal of Global Optimization. In press.

[21] Z. Opial, "Weak convergence of the sequence of successive approximations for nonexpansive mappings," Bulletin of the American Mathematical Society, vol. 73, pp. 591-597, 1967.

[22] W. Takahashi, Nonlinear Functional Analysis, Yokohama Publishers, Yokohama, Japan, 2000.

[23] M. O. Osilike and D. I. Igbokwe, "Weak and strong convergence theorems for fixed points of pseudocontractions and solutions of monotone type operator equations," Computers $\mathcal{E}$ Mathematics with Applications, vol. 40, no. 4-5, pp. 559-567, 2000. 
[24] H. K. Xu, "An iterative approach to quadratic optimization," Journal of Optimization Theory and Applications, vol. 116, no. 3, pp. 659-678, 2003.

[25] H.-K. Xu, "Viscosity approximation methods for nonexpansive mappings," Journal of Mathematical Analysis and Applications, vol. 298, no. 1, pp. 279-291, 2004.

[26] E. Blum and W. Oettli, "From optimization and variationnal inequalities to equilibrium problems," Mathematics Students, vol. 6, pp. 117-136, 2005.

[27] P.-E. Maingé, "Strong convergence of projected subgradient methods for nonsmooth and nonstrictly convex minimization," Set-Valued Analysis, vol. 16, no. 7-8, pp. 899-912, 2008. 